\title{
La formación de las bibliotecarias y las bibliotecas de mujeres en España
}

\author{
Ana M. MUÑOZ-MuÑOZ \\ Instituto Universitario de Investigación de Estudios de la Mujeres y de Género \\ Universidad de Granada. Departamento de Información y Comunicación \\ anamaria@ugr.es \\ Montse ARGENTE JIMÉNEZ \\ Institut Català de les Dones. Centro de Documentación \\ margente@gencat.cat
}

Recibido: Enero 2015

Aceptado: Abril 2015

Resumen: Desde finales del siglo XIX, las mujeres en España acceden a la formación de bibliotecaria, y a partir del XX se crean las primeras bibliotecas de mujeres. Se pretende investigar el proceso de feminización en esta profesión durante este periodo, y su posible influencia en la creación de bibliotecas de mujeres. Para ello, se presenta un recorrido histórico de la formación de las mujeres en la biblioteconomía, tanto en la enseñanza reglada como en los cursos de formación impartidos por instituciones españolas de diversa índole, así como la aparición e importancia progresiva que han venido adquiriendo estas bibliotecas a partir de entonces. Se constata, que aunque ambos recorridos se desarrollaron de manera independiente, la formación de bibliotecarias propició de forma indirecta la creación de bibliotecas de mujeres.

Palabras clave: Bibliotecarias; Bibliotecas de Mujeres; España; Formación.

\section{Women librarians' training and Women’s Libraries in Spain}

\begin{abstract}
Since the end of the 19th century, women have accessed library training and in the start of the 20th century, first women's libraries appear are created in Spain. This study addresses access of women to library training, feminizing the profession and influencing the creation of women's libraries. In order to do so, a review of recent history is performed. On the one hand, analyzing women's training in Higher Education and in programs developed by different Spanish institutions. On the other hand, analyzing the creation and the increasing importance women's libraries have had. Although these two topics had independent developments, it becomes clear that women's inclusion in library studies training fostered the creation of women's libraries.
\end{abstract}

Keywords: Spain; Training; Women librarians; Women’s libraries. 


\section{INTRODUCCIÓN}

A principios del siglo XIX, las bibliotecas existentes en España eran privadas o de uso restringido, perteneciendo principalmente a monasterios, universidades, bibliófilos, nobles, eruditos y palacios reales. De la misma manera, las bibliotecas de las academias, los círculos culturales, los museos, las sociedades económicas, las cámaras y las escuelas de comercio, y los consulados, con fondos bibliográficos de carácter profesional más o menos laico, reservaban el derecho de lectura casi siempre a los socios. En todos estos casos, las bibliotecas no desempeñaban un papel central sino de respaldo, es decir, eran imprescindibles para coleccionar y conservar los documentos en ellas depositados más que centros públicos de acceso a la información (Bartolomé Martínez, 1989). Posteriormente, tras las Leyes desamortizadoras de Mendizábal (1835-36), se crearon las bibliotecas públicas provinciales cuya misión fue recoger todo el patrimonio bibliográfico y documental incautado a las órdenes religiosas suprimidas. No obstante, la biblioteca pública tal como hoy la conocemos, se consolida durante la II República (Martínez Rus, 2003). Recordemos que la población alfabetizada en España en 1860 era de un $20 \%$ (9\% de mujeres y 31\% de hombres) y para 1900 era de un 33\% (25\% de mujeres y 42\% de hombres) (Gabriel, 1997). La biblioteca pública o popular se identificará con un servicio de lectura colectiva, de acceso libre para hombres y mujeres, como una agencia de educación para el pueblo, complementaria de la escuela (Gómez Hernández, 1993).

El desarrollo de éste y otros tipos de bibliotecas va a necesitar personal formado, y para ello se crearán Centros de enseñanzas de biblioteconomía dirigidos a mujeres para cubrir puestos de trabajo con personal especializado en bibliotecas populares como es el caso de la Escuela de Barcelona (1918), personal especializado en investigación para la Escuela del Consejo Superior de Investigación Científica (1944) y en bibliotecas universitarias como la de la Universidad de Navarra (1967).

Es a partir del siglo XX con la aparición de la primera Escuela de bibliotecarias y de la primera Biblioteca para mujeres, cuando podemos iniciar nuestro interés en la línea de investigación "Mujeres y bibliotecas". La bibliografía más completa al respecto, se refiere a aquellas instituciones de enseñanza y a las bibliotecas que aún continúan en funcionamiento como son los casos de la Facultad de Biblioteconomía de la Universitat de Barcerlona (Estivill Rius, 1972, 2006) y la Biblioteca Francesca Bonnemaison (Cabó i Cardona, 1995; Marín Silvestre, 2004; Segura-Soriano, 2007). En el resto de la bibliografía que se ha utilizado a lo largo de este estudio, localizamos referencias puntuales y dispersas sobre la enseñanza de la biblioteconomía dirigida exclusivamente a mujeres y/o bibliotecas de mujeres, así como escasos datos cuantitativos. En la literatura revisada sobre la formación del bibliotecario y la historia de las bibliotecas en España, pasa desapercibida la existencia de las mujeres en estos establecimientos. 
Es por ello interesante recoger y mostrar en un único estudio aquellos centros de enseñanza y bibliotecas de mujeres que por su desaparición, y en algunos casos por su efímera existencia, han caído en el olvido, dando una visión lo más exhaustiva posible de la historia de la formación de las bibliotecarias y de las bibliotecas de mujeres en España.

Así pues, se presenta un recorrido histórico tanto de la formación de las mujeres en biblioteconomía en la enseñanza reglada y en los cursos de formación impartidos por instituciones españolas de diversa índole, como de la creación e importancia que progresivamente han ido adquiriendo las bibliotecas de mujeres. Mediante esta reconstrucción histórica de dos recorridos independientes pero a su vez interrelacionados, este trabajo pretende conocer si el hecho de que las mujeres pudieran acceder a la formación de bibliotecarias, y la consiguiente feminización de la profesión, influyó en la creación de bibliotecas de mujeres.

\section{CONTEXTO HISTÓRICO Y SOCIAL}

La incorporación de las mujeres a la formación como bibliotecarias y al trabajo en las bibliotecas españolas es más tardía con respecto a los hombres, tanto porque el acceso de las mujeres a la educación escolar no se regulariza hasta 1857 con la Ley de Instrucción Pública (Scanlon, 1987), como por su tardía incorporación a los estudios de Bachillerato en 1874, que además inicialmente no tenían efecto profesional, sólo académico. De la misma forma ocurre con el acceso a la educación superior universitaria mediante el Real Decreto de 8 de marzo de 1910. Aunque las mujeres puedan ya acceder legal y libremente a la universidad, el contexto social, condicionado por factores ideológicos, culturales y económicos hacen que su presencia sea minoritaria, y que esta se produzca solamente en aquellos tipos de carreras consideradas socialmente más adecuadas para la población femenina. A pesar de ello, esta incorporación a la formación facilitará la creación de nuevas profesiones en el sector servicios como mecanógrafas, telefonistas, enfermeras o bibliotecarias, y su integración en la administración pública. Todo ello aportará nuevas salidas profesionales en las primeras décadas del siglo XX y el acceso a carreras de profesiones liberales, pero principalmente a ocupaciones docentes (Flecha García, 1996). La enseñanza era considerada un trabajo femenino aceptado y socialmente reconocido. La identificación de la docencia con el trabajo bibliotecario, siguiendo los mismos cánones, provocará que éste también se feminice rápidamente.

Por otro lado, la creación de Bibliotecas de Mujeres en España a principios del siglo XX, favoreció el acceso a la educación, constituyendo el origen de centros que ya en los años ochenta tendrán como objetivo principal fomentar la igualdad entre mujeres y hombres. 
Figura 1. Eventos de formación de las bibliotecarias y las bibliotecas de mujeres en España

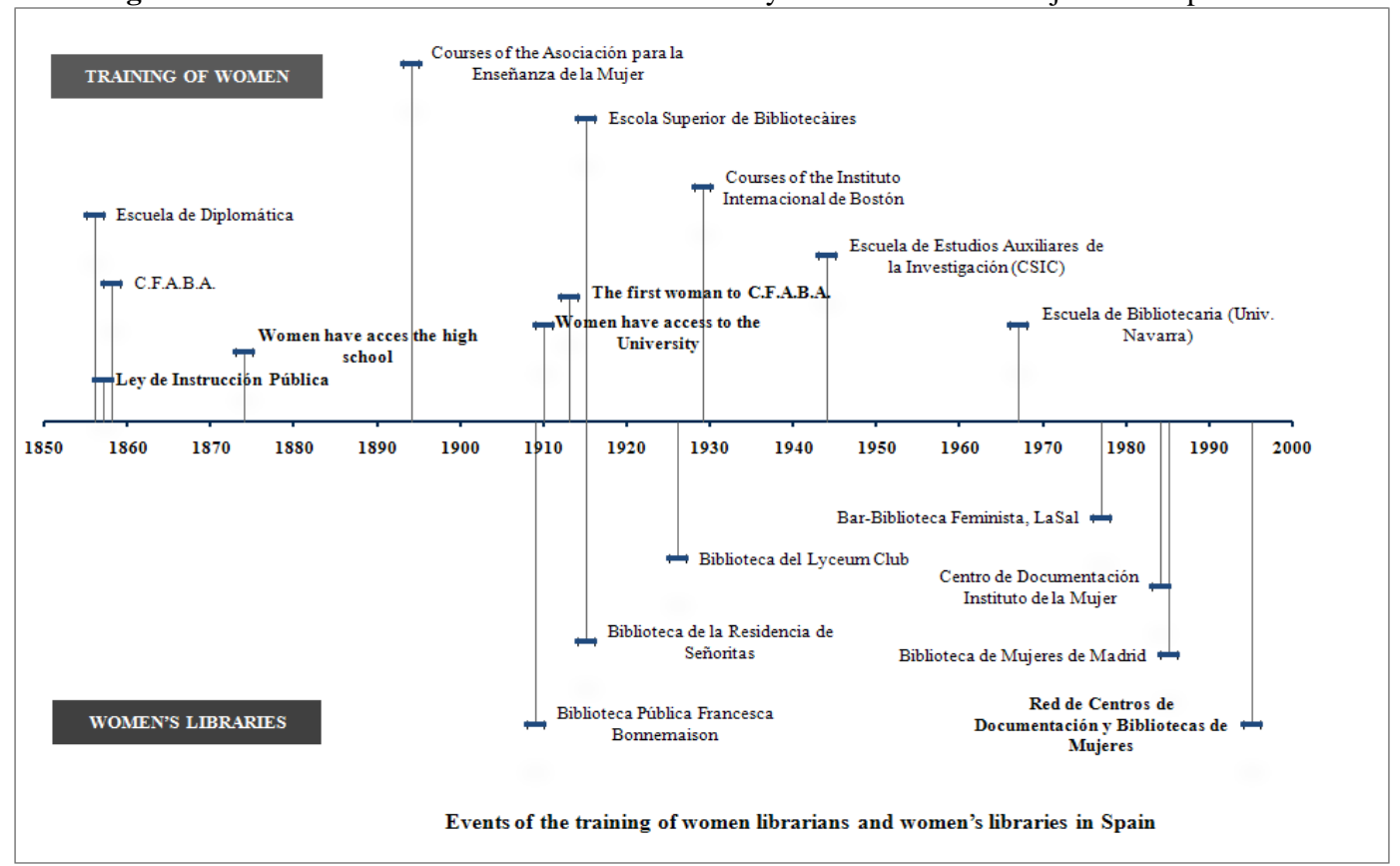

(Elaboración propia)

\section{FORMACIÓN DE BIBLIOTECARIAS}

La formación de bibliotecarios en España tiene su punto de partida en 1856 con la creación de la Escuela de Diplomática ${ }^{1}$, dos años más tarde mediante el Real Decreto de 17 de julio de 1858, se crea el Cuerpo Facultativo de ArchiverosBibliotecarios ${ }^{2}$. Para ingresar en el mismo era necesario haber obtenido el título de archivero-bibliotecario expedido por la Escuela Superior (Escuela Superior de Diplomática, 1865), aunque las mujeres al no tener acceso al Título de Bachiller, tampoco podían acceder a la Escuela Superior.

En 1871 se constituye en Madrid la Asociación para la Enseñanza de la Mujer con el fin de fomentar "la instrucción y educación de la mujer en todas las esferas y condiciones de la vida social”. Fundada por Fernando de Castro, uno de los

${ }^{1}$ Con la Ley de Instrucción Pública (Ley Moyano), que le concede el rango de "superiores" a sus estudios, pasa a llamarse Escuela Superior de Diplomática.

2 Algunos años más tarde sería el Cuerpo Facultativo de Archiveros, Bibliotecarios y Anticuarios, para terminar llevando el nombre de Cuerpo Facultativo de Archiveros, Bibliotecarios y Arqueólogos. 
seguidores del Krausismo ${ }^{3}$ más progresistas, albergaba a varias Escuelas de Primaria, de Segunda Enseñanza, de Institutrices o de enseñanza superior, la Preparatoria para el ingreso a la Escuela Normal Central de Maestras, la Escuela o curso de Comercio, y la de Correos y Telégrafos (Ballarín Domingo, 1989; Flecha García, 1996). Además, ofertaba clases especiales de idiomas, dibujo, pintura, música y corte y confección. Entre ellas, en 1894 figuraban las clases especiales de bibliotecarias y archiveras, con una duración de dos cursos de ocho meses cada uno. Las consideraciones que hace la Asociación para impartir dichas clases se fundamentaban en el reducido número de alumnos que cursaban la carrera en la Escuela Superior de Diplomática, finalizando por término medio al año 12 jóvenes, no siendo éstos suficientes para cubrir las necesidades de los Archivos y Bibliotecas del Estado (Proyecto Archiveras y Bibliotecarias, 1894). De esta manera se inicia la posibilidad de acceso de las mujeres al trabajo bibliotecario. A esta consideración muy práctica y objetiva para que las jóvenes se incorporen al mundo laboral, le sigue otra que asigna a las chicas los estereotipos sexuales propios o característicos de la época calificándolas de ordenadas, minuciosas, pacientes, limpias y escrupulosas, y que recogemos textualmente:

Inútil es decir que las tranquilas funciones de Archiveros y Bibliotecarios, en el recogimiento y sosiego de los establecimientos de su cargo, son muy a propósito para la mujer y están muy en armonía con su aptitudes, puesto que la ordenación y arreglo de Archivos y Bibliotecas exigen principalmente la minuciosidad, paciencia, orden y aseo que de ordinario predominan en el carácter del sexo. Para la copia de documentos paleográficos, por ejemplo, siempre será más escrupulosa la mujer que el hombre. (Proyecto Archiveras y Bibliotecarias, 1894)

En 1900 desaparece la Escuela Superior de Diplomática, al integrarse las enseñanzas que impartía en la Facultad de Filosofía y Letras de la Universidad de Madrid. A partir de este momento y hasta la creación de las escuelas universitarias de Biblioteconomía y Documentación, en 1983, no hay un intento oficial de institucionalizar una formación específica en este tema. (García Ejarque, 1993; Orera Orera, 2002)

${ }^{3}$ El krausismo es una doctrina que defiende la tolerancia académica y la libertad de cátedra frente al dogmatismo. Debe su nombre al pensador postkantiano alemán Karl Christian Friedrich Krause (1781-1832). Esta filosofía tuvo gran difusión en España a mediados del XIX donde alcanzó su máximo desarrollo práctico, gracias a la obra de su gran divulgador, Julián Sanz del Río y a la Institución Libre de Enseñanza dirigida por Francisco Giner de los Ríos, además de la contribución de un gran jurista como Fernando de Castro. Se da por acabado el krausismo en 1939, con el final de la II República española. 
La publicación de dos normativas en 1910, una que establecía condiciones de igualdad con los hombres para acceder a estudios universitarios y otra que reconoce el derecho de las mujeres, una vez licenciadas, a presentarse a las oposiciones convocadas por el Ministerio de Instrucción Pública y Bellas Artes, propició una situación positiva que se dejó notar en todas las universidades (Flecha García, 1996). Hasta ese momento los títulos de licenciatura obtenidos por mujeres no habían sido válidos para ocupar ningún puesto en las administraciones públicas; ahora desaparecían tales limitaciones. Una de las Facultades con mayor número de alumnas es la de Filosofía y Letras debido a la aparición de nuevos campos laborales entre ellos la enseñanza media y superior y el acceso al Cuerpo Facultativo de Archiveros, Bibliotecarios y Arqueólogos (C.F.A.B.A.), trabajo que requería la obtención de dicho título (Muñoz-Muñoz, 2006). Su auge coincide con la apertura a las mujeres de la posibilidad de ejercer la docencia media y superior y actividades afines, como las incluidas en el C.F.A.B.A., al parecer coincidentes con las naturales inclinaciones femeninas. Gregorio Martínez Sierra elogia la figura de la bibliotecaria:

Me parece muy bien... haber pensado en las mujeres para el oficio de bibliotecarias. No le hay más propio para ellas, ni que requiera condiciones más esencialmente femeninas para ejercerle bien: orden, limpieza, paciencia, espíritu de clasificación, estudio de las lenguas. (Martínez Sierra, 1917: 193-194)

El 26 de julio de 1913, Ángela García Rives licenciada en Filosofía y Letras, es la primera mujer que se vincula oficialmente a la profesión de bibliotecaria tras ingresar mediante oposición al C.F.A.B.A. (García Ejarque, 1993; Díaz Andreu, 1998).

A partir de que a las mujeres se les permite opositar al C.F.A.B.A., comienza la feminización de la profesión en todas sus escalas, proceso mediante el cual esta incorporación al trabajo bibliotecario llega a sobrepasar en número y porcentaje a los hombres. Basándonos en la elaboración de los datos de las convocatorias, el número de plazas y el listado de aprobados en las oposiciones ${ }^{4}$, distinguimos tres periodos:

${ }^{4}$ Las fuentes consultadas para conocer los datos de las convocatorias, el número de plazas y listado de aprobados en las oposiciones han sido: Gaceta de Madrid (1697 - 31 de marzo de 1934); Gaceta de Madrid: Diario Oficial de la República (1 de abril de 1934 - 8 de noviembre de 1936), Gaceta de la República: Diario Oficial (10 de noviembre de 1936 - 28 de marzo de 1939), Boletín Oficial del Estado (2 de octubre de 1936 - 27 de febrero de 1961), Boletín Oficial del Estado: Gaceta de Madrid (28 de febrero de 1961 - 23 de julio de 1986) y Boletín Oficial del Estado (24 de julio de 1986 - hasta 1999). 
a) Periodo de despegue, coincidiendo con la República y la Guerra Civil, desde 1912 hasta los años 30. El C.F.A.B.A. convoca 190 plazas y aprueban un 37\% de mujeres. A su vez en el Cuerpo Auxiliar de Bibliotecas, Archivos y Museos creado en 1932 convoca 63 plazas y aprueba el 43\% de mujeres.

b) Periodo de afianzamiento, durante la dictadura Franquista, desde los años 40 hasta 1974. El C.F.A.B.A. convoca 285 plazas de las que el 66\% son aprobadas por mujeres. Y el Cuerpo de Auxiliar convoca 376 plazas aprobadas por un 73\% de mujeres.

c) Periodo de consolidación, a partir de la Transición política española y la democracia, desde 1975 hasta 1999, en el que de 254 plazas convocadas para bibliotecas aprueban el 77\% de las mujeres. En 1976 se hace la última y única convocatoria del Cuerpo de Auxiliar, creándose en 1977 un nuevo Cuerpo el de Ayudantes de Archivos, Bibliotecas y Museos. Para el Cuerpo de Auxiliar se convocan 17 plazas y el 82\% de mujeres aprueban, y para el Cuerpo de Ayudantes 486 plazas aprobando un 79\% de mujeres.

De estos periodos de feminización de las bibliotecas, los dos primeros coinciden con la génesis de las Escuelas de formación profesional de bibliotecarias. El de despegue con la creación de la Escola Superior de Bibliotecàries y la Escuela de bibliotecarias del Instituto Internacional, y en el de afianzamiento se incorporan a las enseñanzas anteriores la Escuela de Bibliotecarias de la Universidad de Navarra y la Escuela de Estudios Auxiliares de la Investigación. El periodo de consolidación corresponde al momento en las que todas estas enseñanzas dejan de ser dirigidas exclusivamente a mujeres, creándose en los años ochenta nuevas Escuelas Universitarias mixtas.

En 1915, dos años más tarde de que Ángela García Rives se incorporara al C.F.A.B.A., la Mancomunidad de Cataluña funda en Barcelona la Escola Superior de Bibliotecàries, siendo la segunda de Europa y la primera y única escuela de bibliotecarias del Estado que ha tenido continuidad hasta hoy en día. Su misión fundamental consistió en preparar a las bibliotecarias de la red de bibliotecas populares que se crearon en Cataluña entre 1914 y 1923. En esta escuela se formó al personal que había de ocuparse de las bibliotecas públicas recién creadas, y su director, Eugeni d’Ors, diseñaba el perfil profesional que éste había de tener justificando la elección de mujeres con argumentos tales como que el personal debía ser forzosamente femenino, por una cuestión de reducción de costes ya que la jornada laboral femenina era más reducida que la de los hombres y, además, no hacía falta pagar sueldos muy elevados a las mujeres ya que la principal aportación a la economía doméstica provenía del marido y ellas sólo representaban una pequeña contribución. A su vez la selección de personal femenino bien escogido aportaría el "carácter atractivo, amable, de limpieza y coquetería que queremos dar a nuestras instalaciones" (Mancomunitat de Catalunya, 1923). También defendía que el trabajo educativo y misionero que debían tener las bibliotecas, correspondía 
más a la idiosincrasia femenina. El proyecto daba una salida laboral y por consiguiente, una cierta independencia a las chicas de clase media, sobre todo a aquellas que procedían de familias intelectuales, que serían las alumnas del centro.

En 1925 esta escuela es integrada en la Escuela Superior de la Mujer, experimentando un cambio de orientación y ofertando hasta 1930, entre otros, los estudios de la Carrera de Bibliotecaria, Archivera y Funcionaria. Ese mismo año, se suprime la Escuela Superior de la Mujer, y la Escola Superior de Bibliotecàries retorna a los planteamientos iniciales viviendo una de sus etapas más brillantes como institución de enseñanza plenamente consolidada. Finalizada la Guerra Civil Española en 1939, pasó a depender de la Diputación provincial de Barcelona con el nombre oficial castellanizado de Escuela de Bibliotecarias hasta que en 1974 pasa a llamarse Escuela de Bibliología, dejando de ser exclusivamente femenina. Desde su primera promoción de 1918 hasta 1973, el número de diplomadas alcanza 651 (García Ejarque, 1993). Si tenemos presente que cada año terminaban los estudios menos de 12 bibliotecarias, podemos comprender que la finalidad de la Escuela de la Diputación era básicamente formar profesionales para sus bibliotecas, de hecho para presentarse a la oposición en sus convocatorias se requería el titulo de la Escuela de Bibliotecarias. En 1982 es adscrita a la Universitat de Barcelona convirtiéndose en la Escola Universitària Jordi Rubió i Balaguer de Biblioteconomia i Documentació hasta el curso 1997-98 en que se produce su plena integración como Facultad en la Universitat de Barcelona (Estivill Rius, 2006; Mayol, 2002).

A la imagen de la Escola Superior de Bibliotecàries, en 1967 el romanista Álvaro d'Ors, hijo del fundador de la misma, crea en la Universidad de Navarra una Escuela de Bibliotecarias de carácter privado, siendo su directora Nuria Orpi Prat, bibliotecaria diplomada por la Escola Superior de Bibliotecàries y miembro del C.F.A.B.A. Esta Escuela sólo permanecería en funcionamiento hasta 1977 (Orpi, 1973). A diferencia de la Escola de Barcelona, esta nació como institución universitaria, motivado por la escasez de personal que prestaba servicios auxiliares y administrativos en las Bibliotecas de la Universidad de Navarra y a la creación del Servicio Bibliotecario. En el verano de 1968 algunos periódicos dan la noticia de la aparición de la nueva Escuela, insistiendo en la importancia de la formación del personal técnico de las bibliotecas, y haciendo también referencia a las cualidades que se requerían para ser una buena bibliotecaria:

... una inteligencia intuitiva, muy propia de la mujer, que le permita adivinar lo que materialmente no ha podido aprender de todo el conjunto de las ciencias; luego una comprensión profunda del trabajo científico a fin de facilitarlo y no dificultarlo; un sentido del orden que compense el inevitable desorden que puede producir el trabajo intenso de los hombres de ciencia; por último, la capacidad de trabajar en equipo con todo el personal bibliotecario. Y podrían quizá 
añadirse la curiosidad intelectual permanente, la paciencia y el don de gentes. (Orpi, 1973: 50)

Desde su inicios en 1968 hasta el curso 1971/1972, se diplomaron 58 mujeres que se incorporaron al Servicio de Bibliotecas de la Universidad de Navarra así como a otras bibliotecas populares, de corporaciones y de empresas. (Mateu Ifars, 1981).

Salvo la excepción de la Escola de Barcelona, no hay en España hasta los años 40 centros educativos que impartiesen enseñanzas especializadas en biblioteconomía con una duración significativa. No obstante es imprescindible mencionar experiencias como la Escuela de bibliotecarias del Instituto Internacional de influencia estadounidense. El Institute International for Girls (IIG), más conocido en España como el Instituto Internacional de Bostón, instalado en Madrid desde 1909, fue fundado en 1892 por Alice Gordon Gulick y sus colaboradores para alcanzar, a través de la libertad de conciencia y de la educación de las mujeres, una emancipación social y cultural de las mismas, que presentara al pueblo español un nuevo ideal femenino y que con él se consiguiera, en la práctica, la participación de las mujeres españolas en la vida del país. El Instituto, siempre alerta a los vacíos en la educación española, organizó los primeros cursos de biblioteconomía para preparar bibliotecarias que sirviesen al público lector e investigador.

En 1929, la bibliotecaria norteamericana Mauda Polley impartió un cursillo sobre el Sistema de Clasificación Decimal de Dewey. Más adelante ese cursillo se convirtió en un programa de dos años de duración de estudios bibliotecarios, de contenidos eminentemente prácticos. Las estudiantes que lo completaban recibían un certificado emitido conjuntamente por la Residencia de Señoritas y el Ministerio de Instrucción Pública. El certificado no era un título oficial de bibliotecaria, ya que para esto era necesario hacer la licenciatura de Filosofía y Letras y completarla con unos estudios más amplios (Zulueta, 1992). Estos cursos fueron durante mucho tiempo los más prestigiosos que existían en España.

Por último, el 31 de mayo de 1944 se crea en Madrid la Escuela de Estudios Auxiliares de la Investigación dependiente del Consejo Superior de Investigaciones Científicas (C.S.I.C.). Su misión fundamental fue la formación de Auxiliares para sus tres funciones: Secretarías, Bibliotecas y Laboratorios. Las Auxiliares de Bibliotecas se formaban en "la técnica bibliográfica, con toda su amplitud de organización de bibliotecas, intercambio, préstamos, fotocopias, catalogación y cuanto da, a la diversidad de bibliotecas dispersas en los Centros de trabajo, una unidad funcional: idiomas, exigencia ineludible de la tarea investigadora en todos sus grados y rutas”.

5 "DECRETO de 31 de mayo de 1944 por el que se crea la Escuela de Estudios Auxiliares de la Investigación, dependiente del Consejo Superior de Investigaciones Científicas”, in Memoria de la Secretaría General: Año 1944 (Madrid: Consejo Superior de Investigaciones Científicas, 1945), 456-457. 
La Escuela fue dirigida por Amadeo Tortajada Ferrandis que a su vez era el director de la Biblioteca General del Consejo. Era ésta una escuela femenina en la que se formaban tanto las llamadas Laborantes, para trabajos en laboratorios, como las Auxiliares de bibliotecas en su Sección de Bibliografía, que estudiaban Biblioteconomía y otras asignaturas específicas, con el fin de trabajar en los Servicios Bibliográficos de la Biblioteca General del C.S.I.C. o en las de sus Institutos de Madrid. Para acceder a esta Escuela se requería Bachiller Superior y un examen de ingreso. Al principio, los cursos tenían una duración de dos años, posteriormente de tres y acababan con un examen final. A partir de la primera promoción de estos cursos, el C.S.I.C. convocaba plazas para la escala de auxiliares a las que se podían presentar las tituladas de la Escuela de Estudios Auxiliares de la Investigación del C.S.I.C. En esta Escuela se titularon 217 alumnas como auxiliares de bibliotecas y el 85\% cubrieron plazas en los servicios bibliotecarios del C.S.I.C. (Tortajada, 1974). La Escuela se cerró en 1973 debido a la pérdida de privilegios que suponía obtener sus titulaciones para entrar en el C.S.I.C., ya que en los últimos años cualquier licenciado podía presentarse a las oposiciones aunque no hubiera pasado por la Escuela.

A lo largo de este recorrido, mientras que las mujeres se forman para ejercer la profesión de bibliotecarias, de manera simultánea se comienzan a establecer bibliotecas para mujeres.

\section{BIBLIOTECAS DE MUJERES}

Las Bibliotecas de Mujeres aparecen en España, al igual que en el resto de Europa en el siglo XX . Algunas autoras identifican estos centros con las mismas generaciones u olas por las que ha pasado el feminismo: bibliotecas de primera y segunda generación, y centros de documentación creados por lo que se ha venido a denominar "feminismo de Estado" (Moller; Nielsen, 1995; Wedborn, 1995). Las bibliotecas correspondientes a la primera generación se crean a comienzos del siglo XX por mujeres pertenecientes a grupos y movimientos sufragistas y pro de sus derechos. En la llamada segunda generación surgida después de la II Guerra Mundial y consolidada a partir de los años 60, se crean bibliotecas de mujeres en gran parte de los países occidentales, aunque España por circunstancias políticas se incorporará a este movimiento ya en la década de los 80 .

Si bien en Europa las primeras bibliotecas para mujeres se crean vinculadas a los movimientos sufragistas o de los derechos de las mujeres, en España la biblioteca pionera nace con una necesidad distinta que es elevar el nivel cultural de las trabajadoras. Las bibliotecas de mujeres estaban ligadas a los proyectos intelectuales del feminismo social en sus dos vertientes, laica y católica. Estas corrientes promovían la educación de las mujeres, su incorporación al mercado laboral -siempre en trabajos considerados socialmente apropiados-, la defensa de la maternidad biológica y social, y un "rearme moral" mediante el concurso del elemento femenino. A partir de la década de los años 80, gran parte de las 
bibliotecas de mujeres españolas que se crean hay que encuadrarlas dentro de lo que se denomina el "feminismo de Estado" o feminismo institucional, pues aparecen por iniciativa de los organismos administrativos que promueven la igualdad de oportunidades entre mujeres y hombres (Torres Ramírez; MuñozMuñoz, 2000).

La Biblioteca Pública Francesca Bonnemaison, creada en Barcelona en el año 1909, probablemente fue la primera biblioteca de mujeres y para mujeres que hubo en Europa. Su objetivo primordial era promover el acceso a la educación y la cultura de las mujeres. Además del servicio de biblioteca general y circulante para las obreras, aunque la entrada era libre para todas las mujeres, sus estatutos preveían impartir clases que combinarían conocimientos científicos, artísticos y manuales para fomentar el bienestar moral y material (Segura Soriano, 2007). Actualmente la Biblioteca Francesca Bonnemaison es de titularidad pública y forma parte del Consorci de Biblioteques de Barcelona, aunque sigue manteniendo y aumentando su fondo especial sobre mujeres.

El marco dual laico y católico del feminismo social de principio de siglo, propició la creación de redes culturales, fomentó el asociacionismo, la gestación de determinados movimientos sociales vinculados a la paz y, ya en los años veinte, la lucha sufragista, la consolidación de sindicatos específicamente femeninos y el movimiento intelectual de «las modernas» (Mangini González, 2001). Íntimamente ligado a éste, tanto que podría considerarse causa y efecto a la vez del mismo, fue la fundación en Madrid de la Biblioteca de la Residencia de Señoritas. La institución que la alberga, la Residencia de Señoritas, se crea en octubre de 1915. Dirigida por María de Maeztu fue "la primera institución oficial con carácter universitario establecida en España para mujeres graduadas". Se preocupaba de la formación integral de las jóvenes, combinando para ello los aspectos intelectual y moral. En estos años la población femenina que cursaba estudios superiores en Madrid era escasa, contabilizando 65 muchachas que estudiaban en la Universidad como alumnas oficiales y 43 como libres (Vázquez Ramil, 2001).

La Residencia vio rebasada su capacidad por la creciente demanda de plazas por lo que fue imprescindible ampliar el espacio, y gracias a la colaboración y el apoyo del International Institute for Girls (IIG), trasladó algunas de sus dependencias (Dirección, Secretaría y Clases) al edificio del IIG. Ambas instituciones unieron sus fondos bibliográficos llegando así a reunir más de doce mil volúmenes, abriendo la biblioteca, que estaba regida por personal americano y español, a todas las mujeres que desearan frecuentarla. A partir de 1928 la organización de la biblioteca contaba con los excelentes y abundantes fondos del IIG, que además de ceder los libros y sus instalaciones, costeó los salarios de algunas bibliotecarias (Vazquez Ramil, 2001). El uso de la biblioteca por las residentes fue de unos dos millares por mes: 
Este solo dato indica que la Biblioteca es el recinto preferido por las alumnas de la casa y que los cuatro mil libros que la forman, cuidadosamente seleccionados oyendo la opinión de las personas especializadas en cada materia, son utilizados por nuestras alumnas tan asiduamente que forma la base principal de su cultura. (Zulueta; Moreno, 1993: 161)

En 1928-29 la directora de la biblioteca Mauda Polley, ayudada por Phella Phipps, inicia la clasificación de los libros siguiendo el sistema decimal de Dewey e imparte clases de Biblioteconomía muy concurridas por jóvenes deseosas de labrarse un porvenir rápido y bien considerado. Su labor es continuada en años sucesivos por Ruth Hill y Frances Bacon. A partir de 1931 la directora de la biblioteca es Enriqueta Martín, que cuenta con la eficaz colaboración de Nora y Louise Sweeney, y un pequeño grupo de residentes españolas.

En 1933 la Biblioteca de la Residencia de Señoritas, una vez instalada en el edificio del IIG, poseía quince mil volúmenes con obras de consulta, libros de literatura inglesa, norteamericana y francesa, biografías, obras de crítica, teatro, etc. Tuvo un amplio horario de apertura, de nueve de la mañana a nueve de la noche, se consultaron 27.633 obras y disponía de servicio de préstamo (Torres Santo Domingo, 2007).

A partir de 1940, la Residencia de Señoritas, rebautizada con el nombre de Colegio Mayor "Teresa de Cepeda”, fue reinaugurada con una nueva directora Matilde Marquina, la cual se puso en contacto con Susan Huntington Vernon, como representante más significada del IIG, y le manifestó la intención del gobierno español de mantener los viejos y ventajosos vínculos con el IIG. Como enlace actuó la bibliotecaria Enriqueta Martín, que volvió a encargarse de la ordenación de fondos bibliográficos y de impartir cursillos de Biblioteconomía (Vazquez Ramil, 2001). Tras la Guerra Civil, la biblioteca pasa a formar parte de la herencia de la Biblioteca de la Universidad Complutense de Madrid.

A la Biblioteca de la Residencia de Señoritas le siguió la Biblioteca del Lyceum Club. El Lyceum Club Femenino de Madrid se funda en 1926 siguiendo las directrices de los ya existentes en otros países europeos y de acuerdo con el reglamento internacional de los mismos. El Lyceum, en cuyos cuadros dirigentes se integra la avanzadilla más calificada del feminismo español, se encontraba dividido en seis secciones: Social, cuyo fin es lograr la reforma del Código en aquellas leyes que otorgan a la mujer un trato distinto al del hombre en las mismas circunstancias; Música, Artes Plásticas e Industriales, Literatura, Ciencia e Internacional (Capel-Martínez, 1992: 73). En 1929 alcanzó la cifra de 450 socias, unidas bajo el objetivo de «defender los intereses morales y materiales de la mujer, encauzando y desarrollando todas aquellas iniciativas y actividades de índole exclusivamente económica, benéfica, artística, científica y literaria que redunden en su beneficio». Pronto se convertiría en un punto de encuentro de mujeres cultas 
donde se podía discutir sobre la problemática femenina y cultural, impartir charlas y conferencias o estudiar todo tipo de materias, ya que gracias a la financiación de las socias se adquirió una excelente biblioteca. Fue significativa la labor que desarrollaron las distintas secciones o comisiones de estudio desde donde se pidió la revisión del Código civil en aras de la igualdad legal o la educación de las mujeres. Su carácter aconfesional les acarreó críticas públicas por parte de la iglesia, junto a la recomendación de no afiliarse a este tipo de asociaciones (Franco Rubio, 2004; Fagoaga, 2002).

Poco más se sabe de la Biblioteca, salvo que era excelente y que cubría una parte de los objetivos culturales del Lyceum. En un principio fue dirigida por María Lejárraga hasta 1931, a partir de entonces se encarga de esta tarea María Martos de Baeza, socia gestante del Lyceum. María Martos contribuyó a que durante años se organizaran eficazmente conferencias de arte, literatura, veladas musicales, cursos específicos, exposiciones y subgrupos de trabajo sobre la problemática de la mujer. La biblioteca tuvo un gran valor simbólico por lo que significaba de cambio en la relación con la cultura de estas mujeres, frente a sus contemporáneas ancladas en un mundo sin espacio para el desarrollo intelectual (Melián, 2001). Tras la proclamación de la II República (1931-1939), el Lyceum se politizó considerablemente, convirtiéndose en un foro para las mujeres liberales y socialistas progresistas. Se disolvió en 1939, fecha en la que el edificio en que se ubicaba pasa a ser la sede de Falange Española, vendiéndose a muy bajo precio sus instalaciones, en especial la biblioteca (Castillo Marín, 2000).

La Guerra Civil y la dictadura franquista truncaron la trayectoria de la cultura letrada femenina, a la que se habían incorporado escritoras, lectoras y artistas del espacio intergeneracional comprendido entre los años 1914 y 1939. Hasta la Transición política no se dieron las condiciones para la creación de una nueva red de bibliotecas de mujeres. La nueva coyuntura propició la apertura de los primeros centros de documentación vinculados a las Administraciones públicas, a los Seminarios e Institutos Universitarios de Estudios de las Mujeres, y al propio movimiento feminista. Desde la creación de la Biblioteca Francesca Bonnemaison pasaran más de 70 años hasta que a mediados de la década de los 80 , nazcan en España los centros de información y documentación al amparo de esta nueva situación política, y del interés de las instituciones como receptoras de una demanda social para erradicar cualquier forma de discriminación por razón de sexo, y con el objetivo de fomentar la equidad entre mujeres y hombres.

Naciones Unidas declara 1975 Año Internacional de la Mujer y en España se suceden múltiples acontecimientos que coincidiendo con el cambio político, marcarán el inicio de estas unidades de información. Ese mismo año se celebran las Jornadas Nacionales por la Liberación de la Mujer, y un año más tarde las Jornadas de Liberación de la Mujer en Madrid, y en Cataluña las I Jornades Catalanes de la Dona, todas ellas impulsoras y definitorias del movimiento feminista y asociativo del país. 
Por otra parte, la Constitución de 1978 eliminaba definitivamente las desigualdades jurídicas de la legislación y prohibía la discriminación por razón de sexo, ese mismo año el primer gobierno democrático estableció la Subdirección de la Condición Femenina, a partir de la cual se crearía por Ley 16/1983 de 24 de octubre el Instituto de la Mujer. Una vez consolidado el "Estado de las Autonomías", los respectivos gobiernos autonómicos iniciaron acciones políticas que apuntaban a garantizar la igualdad de oportunidades para las mujeres, y que culminaron también en la creación de organismos específicos al respecto.

En las correspondientes leyes de creación de todos estos organismos gubernamentales de naturaleza estatal o autonómica aparece, de forma preceptiva, la creación de servicios de información y documentación o bibliotecas cuyos objetivos primordiales serian promover y satisfacer las consultas que se les planteen sobre cuestiones relativas a mujeres y género, difundir información, documentación y publicaciones de interés para este colectivo y dar apoyo documental a las actividades y funciones de los organismos en los que están integrados.

Así es como hasta la Transición democrática no se dieron en España las condiciones políticas apropiadas para el desarrollo de la llamada segunda generación de bibliotecas de mujeres. Se trata de unidades de recursos y servicios de información especializados que en base a su vinculación orgánica vamos a clasificar, definiendo ciertos rasgos específicos de las mismas, según el tipo de entidad de la cual dependen:

a) Centros de documentación o bibliotecas dependientes de organismos gubernamentales, estatal, autonómicos o locales, promotores de políticas públicas de igualdad. Entre los objetivos de estos organismos será prioritaria la creación de un centro de documentación o biblioteca especializada, cuya misión fuese conseguir la participación igualitaria de las mujeres en la sociedad a través de la información. Son centros que presentan el compromiso de generar y transmitir información para que se tenga en cuenta a las mujeres en el espacio público, favoreciendo su participación en los ámbitos jurídico, profesional, económico, social y cultural. Un ejemplo ha sido el Instituto de la Mujer, de carácter estatal, que ha tenido como finalidad primordial, en cumplimiento y desarrollo de los principios constitucionales, la promoción y el fomento de las condiciones que posibiliten la igualdad social de ambos sexos y la participación de las mujeres en la vida política, cultural, económica y social. En su artículo segundo se explicita, entre otras funciones, la de "Recopilar información y documentación relativa a la mujer, así como la creación de un banco de datos actualizado que sirva de base para el desarrollo de las funciones y competencias del Instituto". Para ello se crea su Centro de Documentación que comenzó a funcionar en 1984 con el objetivo de buscar, tratar y difundir documentación sobre las mujeres. Ofrece los servicios propios de cualquier 
centro de documentación. De especial interés es la base de datos de noticias de prensa que contiene más de 200.000 noticias desde el año 1984 hasta la actualidad.

Por lo que respecta a la Administración local, hay que destacar la creación de los centros de información y recursos para mujeres, como resultado del desarrollo de las políticas de género e igualdad implantadas en el Estado español a partir de los organismos de igualdad que las diseñan. Los primeros centros de este tipo aparecen en la década de los 80, y a lo largo del tiempo han ido recibiendo denominaciones tan diversas como "centros de atención a las mujeres", "equipos de atención a las mujeres" o "puntos de información a las mujeres". En general, un centro de información y recursos para mujeres es un servicio cuya finalidad es dar respuesta a las diferentes demandas de información y atención, así como potenciar procesos de autonomía y contribuir a la superación de todas las situaciones de desigualdad de género.

b) Bibliotecas pertenecientes a los centros de investigación universitaria. Durante la década de los 80 las universidades españolas crean seminarios o institutos universitarios de estudios de las mujeres, que funcionan como centros de investigación, dirigiendo trabajos de investigación o de fin de máster, tesis doctorales y desarrollando programas de doctorado o másteres especializados en estudios de género. A la vez, generan publicaciones especializadas y se dotan de centros de documentación para su apoyo documental, por lo que su misión, muy académica en sí, está sujeta al programa o centro universitario al que dan soporte en la investigación. La mayor parte de los fondos que poseen estos centros están ligados a los proyectos y líneas de investigación desarrollados en los propios seminarios e institutos de cada universidad. Las referencias de sus fondos suelen estar integrados en el catálogo general de las bibliotecas universitarias a la que pertenecen, aunque en muchos casos tengan un espacio físico destinados a ellos.

c) Centros de documentación o bibliotecas creadas por organizaciones pertenecientes al movimiento feminista o a las asociaciones de mujeres. Reflejan un marcado compromiso social y un talante reivindicativo, animando a las mujeres a organizarse para la transformación social con los medios que otorga la posesión de información. Son centros que expresan la voluntad de dar servicio y proporcionar recursos a colectivos de mujeres, a ONGs y a los gobiernos, recopilando y diseminando información para reorientar las políticas. Las asociaciones crean fondos documentales sobre y para las mujeres en sus locales. La falta de recursos dificulta la continuidad de estas colecciones que suelen estar gestionadas por voluntariado de la propia asociación, y en la mayoría de casos no ha tenido oportunidad de recibir formación técnica específica. Dentro de este tipo de bibliotecas, y de especial interés, mencionaremos la Biblioteca de Mujeres de Madrid. Se creó en 1985 por iniciativa de la feminista y documentalista Marisa Mediavilla Herreros a partir de su biblioteca particular, proyecto al que pronto se incorporó la escritora Lola 
Robles. La Biblioteca se creó, entre otros fines, para reunir y conservar documentos que otras bibliotecas consideraban que no era necesario conservar como los elaborados por el movimiento feminista. Su colección consta de unos 25.000 volúmenes, además de revistas, tebeos, carteles, chapas, sellos, etc. (Robles, 2001). En noviembre de 2006 se donó al Instituto de la Mujer, aceptando dicho organismo los fines y objetivos de su creación. La Biblioteca de Mujeres es, en sí misma, un testimonio de la lucha de las mujeres, pues se creó en unos momentos de reivindicación del movimiento feminista de España.

Otro centro que por sus características es digno de recordar, aunque su vida fue de apenas dos años, es el Bar-Biblioteca Feminista, LaSal creado en Barcelona el 14 de mayo de 1977. Esta iniciativa innovadora fue emprendida por Carme Cases, Mari Chordà, Maria José Quevedo, Sat Sapaté y Montse Solà. Se ubicaba en la calle de Riereta 8 como un lugar de encuentro de mujeres, universo creativo cultural en femenino y de debate feminista. Fue un espacio con gran significado ya que, por primera vez, las mujeres y las feministas tienen un punto de referencia propio en la ciudad de Barcelona (Nash, 2005), "Se pretendía que la biblioteca, que comienza a funcionar gracias a donativos, y que llegará a contar con 400 volúmenes, se convirtiese en una biblioteca especializada en temas concernientes a la mujer, al estilo de la Biblioteca Marguerite Durand, que ya funcionaba en París" (Plaza, 1998: 285). De allí partiría la idea de publicar la primera Agenda de la Dona en 1978, editada por LaSal Edicions de les Dones,que fue la primera editorial feminista catalana.

Actualmente las bibliotecas y centros de documentación especializados en temas de mujer se encuentran integradas en la Red de Centros de Documentación y Bibliotecas de Mujeres. La Red se crea en 1995 con el fin de servir como medio de comunicación y cooperación entre los establecimientos documentales especializados en Mujer y Género existentes en todo el estado español. La idea de formar una Red surge en el $2^{\circ}$ Encuentro de Centros de Documentación y Bibliotecas de Mujeres (Madrid, 1995). En diciembre de 1993 en el marco de las Jornadas Feministas estatales "Juntas y a por todas” tiene lugar un taller dedicado a la organización de fondos documentales de mujeres donde coinciden algunas profesionales de las bibliotecas de mujeres. Allí se acordó celebrar al año siguiente un primer Encuentro de Centros de Documentación y Bibliotecas de Mujeres, que organizó la Biblioteca de la Mujer de IPES Elkartea en Pamplona. Ese primer Encuentro estatal supuso un intercambio de experiencias entre centros que hasta entonces habían trabajado de manera aislada, sin apenas relación entre los oficiales y universitarios con los autónomos-feministas, y a partir de la siguiente reunión anual se gestó la Red, cuya finalidad, como hemos mencionado, es la coordinación y colaboración permanentes entre sus integrantes.

Desde entonces, la Red de Centros de Documentación y Bibliotecas de Mujeres se ha ido perfilando cada año como un espacio de intercambio de experiencias y 
colaboración que no depende de ninguna entidad administrativa, y en el que actualmente participan unas 40 unidades de información, bibliotecas y servicios de información de diferentes autonomías y ciudades de España, cuya tipología y dependencia orgánica es heterogénea, integrando centros de documentación de los Institutos universitarios de estudios de género, bibliotecas del movimiento feminista y asociativo, centros de información de la administración local y servicios de documentación de organismos gubernamentales promotores de políticas públicas de igualdad (Muñoz-Muñoz, Argente Jiménez, 2010; Argente Jiménez, 2008).

\section{CONCLUSIÓN}

No existen pruebas directas de que las mujeres bibliotecarias propiciaran la creación de bibliotecas de mujeres pero se podría deducir, contextualmente, que tuvieron su influencia. Efectivamente, su acceso a la formación bibliotecaria, a la cultura en general, la preocupación por su propia capacitación formativa y sobre todo su presencia como trabajadoras, gestoras y creadoras de bibliotecas, fue facilitando la creación de vínculos y relaciones profesionales reforzado por su tesón para avanzar frente a una sociedad que las infravaloraba intelectualmente. En consecuencia participaron en la creación de espacios culturales simbólicos que ellas mismas gestionaron y financiaron. Asimismo, contribuyeron al desarrollo intelectual de sus contemporáneas mediante el ejercicio de su enorme vocación profesional, su espíritu de servicio y la confianza del trabajo bien hecho, a pesar de que éste estuviera poco reconocido o mal pagado. Todo ello influiría en un futuro mejor para la cultura en general, las bibliotecas y para las mujeres en particular.

Desde estas premisas, podríamos afirmar que el acceso a las mujeres a la formación como bibliotecarias, que dio lugar a la feminización de la profesión, ha sido un proceso de subversión silencioso y paulatino, llevado a cabo por una mayoría de mujeres que ejercían esta profesión. Este proceso acompañado por los cambios sociales y políticos acontecidos en España principalmente en el último cuarto del siglo XX, propició finalmente la creación de las bibliotecas de mujeres en España.

Tuvieron que pasar muchos años para que la identidad cultural y social de las mujeres se afianzara lo suficiente, dando lugar a un nuevo conocimiento y una nueva cultura de la información con visión femenina. Cultura y conocimiento que desde los centros de documentación, archivos, bibliotecas y otras unidades de información sobre mujeres, se ha contribuido a preservar, potenciar y difundir. Por ello, se debe tener muy presente que estos centros dedicados a conservar el patrimonio cultural elaborado por las mujeres y sobre ellas, son lugares fundamentales para salvaguardar y construir su memoria histórica de cara a futuras generaciones. La existencia de las unidades de información y documentación sobre mujeres han dotado de autoridad al conocimiento elaborado por y sobre las 
mujeres. Como tales organizaciones legitimadoras y difusoras de la actividad que desarrollan, contribuyen a visibilizar los saberes femeninos y a romper con el silencio en torno a las mujeres.

Desde el contacto, la cooperación y las redes que las tecnologías de la información y de la comunicación nos permiten, junto con las alianzas personales y profesionales que se tejen desde las bibliotecas de mujeres, debemos seguir trabajando para empoderar a las mujeres, para reforzar su situación a través de la información, y para mantener viva su historia poniendo al alcance de la sociedad sus documentos, sus archivos y, en definitiva, sus fuentes de información.

\section{REFERENCIAS BIBLIOGRÁFICAS}

ARGENTE JIMÉNEZ, Montserrat (2008). "Bibliotecas de Mujeres", en Anuario de bibliotecas españolas 2008. Málaga: Fundación Alonso Quijano, pp. 302305.

BALLARÍN DOMINGO, Pilar (1989). "La educación de la mujer española en el siglo XIX", en Historia de la Educación, nº 8, pp. 245-260.

BARTOLOMÉ MARTÍNEZ, Bernabé (1989). "La bibliotecas públicas provinciales (1835-1885): un intento de promoción de la lectura en España", en Revista de Educación, $\mathrm{n}^{\circ}$ 288, pp. 271-304.

CABÓ I CARDONA, Ana (1995). "Biblioteca Popular Francesca Bonnemaison, 1909-1995: historia i ús actual", en Item, no 17, pp. 66-73.

CAPEL MARTÍNEZ, Rosa $\mathrm{M}^{\mathrm{a}}$ (1992). El sufragio femenino en la Segunda República Española. Madrid: Horas y horas.

CASTILLO MARTÍN, Marcia (2000). "Contracorriente: memorias de escritoras de los años veinte” [en línea]. Espéculo. Revista de estudios literarios, $\mathrm{n}^{\circ} 17$. Disponible en www.ucm.es/info/especulo/numero17/memor_20.html. [Consulta: 10/06/2014].

DÍAZ ANDREU, Margarita (1998). "Spanish Women in a Changing World", en DÍAZ-ANDREU GARCÍA, Margarita and Marie Louise STIG SØRENSEN (eds) Excavating Women: a history of women in European Archaeology. London: New York: Routledge, pp.125-145

ESCUELA SUPERIOR DE DIPLOMÁTICA -Madrid- (1865). Reglamento de la escuela superior de diplomática: Creada en Madrid por Real Decreto de 7 de octubre de 1856 y confirmada por la ley de instrucción pública de 9 de septiembre de 1857: precedido de una introducción histórica y acompañado de la legislación vigente sobre archivos y bibliotecas. Madrid: Imp. y Est. de M. Rivadeneyra.

ESTIVILL RIUS, Assumpció (2006). "Una mirada retrospectiva: de l’Escola Superior de Bibliotecàries a la Facultat de Biblioteconomia i Documentació (1915-2005)" [en línea], BiD: textos universitaris de biblioteconomia i 
documentació 4. Disponible en: www2.ub.edu/bid/consulta_articulos.php? fichero=16estivi.htm. [Consulta: 10/06/2014].

- (1992). L’Escola de Bibliotecàries: 1915-1939. Barcelona: Diputació.

FAGOAGA, Concha (2002). "El Lyceum Club de Madrid, elite latente ", en BUSSY-GENEVOIS, Danièle (dir). Les espagnoles dans l'histoire. Une sociabilité démocratique (XIXe-XXe siècles). Saint Denis: Presses Universitaires de Vicennes, pp. 145-167.

FLECHA GARCÍA, Consuelo (1996). Las primeras universitarias en España (1872-1910). Madrid: Narcea.

FRANCO RUBIO, Gloria (2004). "Los orígenes del sufragismo en España", en Espacio, Tiempo y Forma, Serie V, Historia Contemporánea, no 16, pp. 455484.

GABRIEL, Narciso de (1997). "Alfabetización, semialfabetización y analfabetismo en España (1860-1991)", en Revista Complutense de Educación, $\mathrm{n}^{0}$ 1, vol. 8, pp. 199-231.

GARCÍA EJARQUE, Luis (1993). La formación del bibliotecario en España. Madrid: ANABAD.

GOMEZ HÉRNADEZ, José A. (1993). "La preocupación por la lectura pública en España: las bibliotecas «populares». De las Cortes de Cádiz al plan de bibliotecas de María Moliner", en Revista General de Información y Documentación, ${ }^{\circ}$ 2, vol. 3, pp. 55-94.

MANCOMUNITAT DE CATALUNYA. Direcció Tècnica de Biblioteques Populars. Consell de Pedagogia (1923). Anuari de les Biblioteques Populars. 1922-1923. Barcelona: Mancomunitat de Catalunya.

MANGINI GONZÁLEZ, Shirley (2001). Las modernas de Madrid: las grandes intelectuales españolas de la vanguardia. Barcelona: Península.

MARÍN SILVESTRE, Dolors (2004). Francesca Bonnemaison: Educadora de ciutadanes. Barcelona: Institut d'Edicions de la Diputació de Barcelona.

MARTINEZ RUS, Ana (2003). La política del libro durante la Segunda República: socialización de la lectura. Gijón: Trea.

MARTÍNEZ SIERRA, Gregorio (1917). Feminismo, Feminidad, Españolismo. Madrid: Renacimiento.

MATEU IFARS, Josefina (1981). "Biblioteconomía en la Universidad", en Boletín de la Anabad, n³1, vol. 4, pp. 637-651.

MAYOL, Carme (2002). "Passat i present de la professió bibliotecària" [en línea] BiD: textos universitaris de biblioteconomia i documentació 9. Disponible en: www.ub.es/biblio/bid/09mayol.htm. [Consultado: 10/06/2014].

MELIÁN, Elvira (2001). "Rastros de nube: María Martos de Baeza y su mundo", Arenal. Revista de historia de las mujeres, $\mathrm{n}^{\circ}$ 8, pp. 379-388.

MOLLER JENSEN, E.; NIELSEN, J. (1995). "Highlighting Women in Mainstream Information: a Danish Center on Women and Gender", en STEINER MOSELEY, Eva (ed.). Women, information, and the future: 
collecting and sharing resources worldwide. Fort Atkinson, Wisconsin: Highsmith Press, pp. 97-100.

MUÑOZ-MUÑOZ, Ana M. (2006). Presencia y producción científica de las profesoras en la Universidad de Granada (1975-1990). Granada: Editorial de la Universidad de Granada.

MUÑOZ-MUÑOZ, Ana M.; ARGENTE JIMÉNEZ, Montse (2010). "Red de Centros de Documentación y Bibliotecas de Mujeres: cooperación entre las bibliotecas feministas españolas", en El profesional de la información, $\mathrm{n}^{\circ}$ 19, vol. 5, pp. 504-509.

NASH, Mary (2005). "El moviment feminista durant la transició", en PAGÈS I BLANCH, Pelai (ed). La transició democràtica als Paisos Catalans: Història i memoria. Valencia: Universitat de València, pp. 335-365.

ORERA ORERA, Luisa (2002). "La evolución en la formación de los bibliotecarios", en Documentación de las Ciencias de la Información, vol 25, pp. 167-188.

ORPI, Nuria (1973). "La Escuela de Bibliotecarias de la Universidad de Navarra", en Boletín de la ANABA, no 23, vol. 1-2, pp. 45-53.

PLAZA, Elsa (1998). "LaSal, bar-biblioteca feminista: un espai per a la utopia", en 20 anys de Feminisme a Catalunya Jornades 24, 25 i 26 de maig de 1996. Barcelona: Associació de Dones per a la Celebració dels 20 Anys de les Primeres Jornades Catalanes de la Dona.

PROYECTO ARCHIVERAS Y BIBLIOTECARIAS (1894). Fondos de la Fundación Fernando de Castro-A.E.M. [en línea]. Disponible en: www.fernandodecastro.org/images/proyecto.pdf. [Consultado: 10/06/2014].

ROBLES, Lola (2001). Biblioteca de Mujeres. Madrid: BdM.

SCANLON, Geraldine M. (1987). "La mujer y la instrucción pública de la ley Moyano a la II República", en Historia de la educación: Revista interuniversitaria, ${ }^{\circ}$ 6, pp. 193-208.

SEGURA-SORIANO, Isabel (2007). Memòria d'un espai: Institut de Cultura i Biblioteca Popular de la Dona 1909-2003. Barcelona: Institut d’Educació de Barcelona; Publicacions de l'Abadia de Montserrat.

TORRES RAMÍREZ, Isabel de; MUÑOZ-MUÑOZ, Ana M. (2000). Fuentes de información para los Estudios de las Mujeres. Granada: Editorial Universidad de Granada.

TORRES SANTO DOMINGO, Marta (2007). "De la Biblioteca de la Universidad Literaria de Madrid a la Biblioteca de la Universidad Complutense", en $\mathrm{M}^{\mathrm{a}}$ GALLEGO RUBIO, Cristina and MÉNDEZ APARICIO, Juan Antonio (ed.). Historia de la Biblioteca de la Universidad Complutense de Madrid. Madrid: Editorial Complutense, pp. 133-168.

TORTAJADA, Amadeo (1974). "Escuela de Estudios Auxiliares de la Investigación (Ayer, hoy y su mañana)", en Arbor, nº 89, pp. 121-131. 
VÁZQUEZ RAMIL, Raquel (2001). La Institución libre de Enseñanza y la educación de la mujer en España: la Residencia de Señoritas (1915-1936) Tesis doctoral, Universidad de La Coruña.

WEDBORN, Helena (1995). "The Women's History Collections: a Documentation Center integrated into a University Library", en STEINER MOSELEY, Eva (ed.). Women, information, and the future: collecting and sharing resources worldwide. Fort Atkinson, Wisconsin: Highsmith Press, pp.151-157.

ZULUETA, Carmen de (1992). Cien años de educación de la mujer española: Historia del Instituto Internacional. $2^{\text {a }}$ ed. Madrid: Castalia.

ZULUETA, Carmen de; MORENO, Alicia (1993). Ni convento ni college: La Residencia de Señoritas. Madrid: Publicaciones de la Residencia de Estudiantes. 